\title{
Variabilidad genética en mamey (Pouteria sapota (JAQ.) H.E. Moore \& Stearn) basada en marcadores moleculares y morfologia de fruto
}

\author{
Genetic variability in mamey (Pouteria sapota (Jaq.) H.E. Moore \& Stearn) based on molecular \\ markers and fruit morphology
}

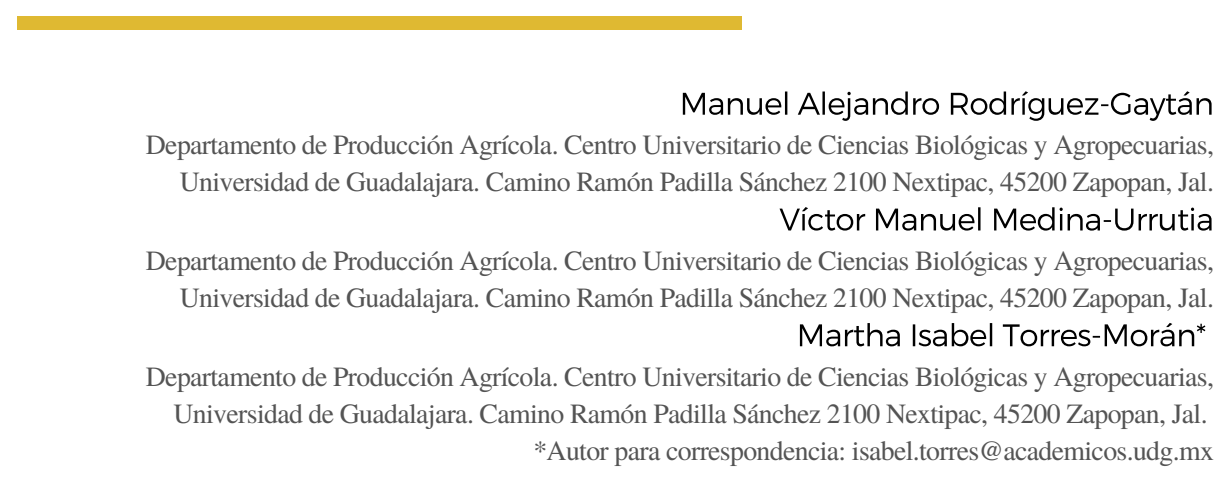

\section{Resumen}

El mamey (Pouteria sapota (Jacq.) H.E. Moore \& Stearn) ha sido un importante fuente de alimento en México y Centroamérica de donde es originario, así como en otras zonas tropicales del mundo donde ha sido introducido. A pesar de la importancia que representa este recurso fitogenético y la variabilidad que ha sido reportada anteriormente, no se han establecidos parámetros de variabilidad y diversidad genética que permitan llevar a cabo planes de desarrollo sustentable y explotación comercial. El objetivo de este trabajo fue determinar la variabilidad genética en una población de mamey del estado de Colima, basada en marcadores moleculares y características del fruto. Se utilizó una muestra de plantas provenientes del estado de Yucatán, como germoplasma de referencia con el origen. Se encontró que existe un alto nivel de variabilidad genética intrapoblacional y un grado considerable de diferenciación en términos de frecuencias alélicas entre las muestras procedentes de Colima y Yucatán, los marcadores ISTR e ISSR demostraron ser útiles en la segregación de las muestras en función de su lugar de origen.

Palabras clave: Estructura genética, Componentes principales, heterocigosidad

\begin{abstract}
The mamey (Pouteria sapota (Jacq.) H.E. Moore \& Stearn) has been an important food source in Mexico and Central America from where it originates, as well as in other tropical areas of the world where it has been introduced. Despite the importance of this phytogenetic resource and the variability that has been previously reported, parameters of genetic variability and diversity have not been established that allow for sustainable development and commercial exploitation plans to be carried out. The objective of this work was to determine the genetic variability in a population of mamey from the state of Colima, based on molecular markers and characteristics of the fruit. A sample of plants from the state of Yucatán was used as reference germplasm with the origin. It was found that there is a high level of intrapopulation genetic variability and a considerable degree of differentiation in terms of allelic frequencies between the samples from Colima and Yucatán, the ISTR and ISSR markers proved to be useful in the segregation of the samples based on their origin.
\end{abstract}

Key words: Genetic structure, principal components, heterozygosity 


\section{Introducción}

El mamey (Pouteria sapota) es una especie frutal que se consume ampliamente en nuestro país. Su zona de origen y diversidad, comprende el sureste de México y Centroamérica hasta Nicaragua, y ha sido introducida enmuchas partes del mundo (Nava-Cruz y Ricker, 2004 y Azurdia, 2006). En la República Mexicana, el mamey se produce en 15 estados en las modalidades de riego y temporal (Arcos-López, 2011), esta especie ocupa un total de 1618 ha cultivadas. En el periodo que abarca de 2013 a 2017 se ha registrado tan solo un aumento de 2 ha en la superficie de producción (SIAP, 2018). A pesar de la importancia del cultivo de mamey, su producción se ha visto limitada por factores como prácticas de colecta por extracción, explotación a menor escala en huertos de traspatio y en sistemas agroforestales, donde se le encuentra asociado a otras especies frutales (Azurdia, 2006; Nava-Cruz y Ricker, 2004). Aunado a lo anterior, el manejo postcosecha presenta dificultades debido a las diferencias que existen en la maduración del fruto y a la susceptibilidad a plagas y enfermedades (Gómez-Jaimes et al., 2012).

A nivel morfológico se ha observado que el mamey presenta una amplia variabilidad en caracteres del fruto. En la mayoría de los estudios realizados, las características con mayores tasas de variación en individuos cultivados dentro y fuera de su zona de origen son: peso total, longitud total, diámetro total, grosor del mesocarpio, color del mesocarpio, número se semillas, grosor de epicarpio y textura del epicarpio (Espinos et al., 2005; Bayuelo-Jiménez et al., 2006 y Gaona-García et al., 2008).

El estudio del mamey, llevado a nivel molecular había sido reportado con amplia variabilidad genética entre individuos y entre poblaciones, donde se había observado que, al igual que las poblaciones silvestres, las poblaciones cultivadas presentaban tasas de polimorfismo mayores a 75\% (Carrara et al., 2004; Arellano-Durán, 2012; Ibarra-Estrada, 2012; Rodríguez-Rojas et al., 2012). Sin embargo, en un estudio realizado recientemente por Martínez-Castillo et al. (2019) ha reportado un cuello de botella en poblaciones del estado de Yucatán. El estudio de la variabilidad genética en mamey debe ser una actividad prioritaria a su explotación, ya que con base al conocimiento de las tasas de variación en sus diferentes niveles se proveerá un marco de análisis confiable que permitirá establecer estrategias de conservación y el desarrollo de variedades con base en el material sobresaliente en alguna de sus características (Azurdia, 2006; Elias-Nascimiento et al., 2008; Rodríguez-Rojas et al., 2012).

A nivel nacional el estado de Colima es el sexto en superficie cultivada y en cuanto a producción anual neta de mamey (SIAP, 2018). No existen datos sobre el estado de la variabilidad genética o morfológica de esta especie en los cultivares de este estado.

Los marcadores moleculares, son en la actualidad una herramienta muy útil para el estudio de la variabilidad genética. Existen muchos marcadores dominantes que puedes ser utilizados en diversas especies vegetales. Los ISTR (Inverse Sequence Tagged Repeat) han demostrado tener la capacidad de detectar un alto porcentaje de polimorfismo, ya que están basados en retrotransposones (Infante et al. 2006, 2007; Torres-Morán et al. 2008; Castellanos-Hernánez et al, 2017). Por otra parte, los ISSR (Inter Simple Sequence Repeats) se han probado en numerosos estudios y son capaces de producir datos confiables para el estudio de diversidad y varibilidad genética. El objetivo de este trabajo fue determinar los niveles de variabilidad genética con el uso de marcadores ISTR e ISSR y mediante características del fruto en una población de mamey cultivada con fines comerciales fuera de la zona de origen (Comala, Colima).

\section{Materiales y Métodos}

Material Vegetal

Se colectaron 30 individuos procedentes de una plantación del municipio de Comala, Colima. La colecta consistió por un lado en tejido foliar de 15 árboles adultos y de 15 plántulas para llevar a cabo un estudio de variabilidad y estructura genética de la población. Por otro lado se colectaron 50 frutos de cada uno de los 15 árboles adultos mencionados anteriormente para llevar a cabo una caracterización morfológica y un Análisis de Componentes Principales (ACP). Se incluyeron ocho muestras de árboles adultos provenientes del estado de Yucatán, como referente de origen.

\section{Caracterización Morfológica}

De los 50 frutos colectados de cada uno de los árboles adultos se llevaron a cabo mediciones de siete variables: peso total, longitud total, diámetro total, número de semillas, grosor de la pulpa en corte longitudinal, grosor de la pulpa en corte transversal y forma del fruto de acuerdo a Villegas y Mora (2008).

\section{Variabilidad Genética}

Se realizó una extracción de ADN usando el protocolo reportado por Keb-Llanes et al. (2002). Para estimar la variabilidad genética se utilizaron dos marcadores: ISTR (Inverse Sequence Tagged Repeat) e ISSR (Inter Simple Sequence Repeat). Los iniciadores usados para la amplificación de los fragmentos ISTR fueron: F1 (5'-GCA CTC CAC CAA GAA TAC C- 3'), F91 (5'- ATA TGG ACT TAA GCA AGC CA-3'), B31 (5' - ATT CCC ATC TGC ACC AAT - 3') y B1 (5'- ATC AGG AAG GTC TGT AAA GC- 3'). Los fragmentos obtenidos fueron separados en geles de poliacrilamida $6 \%$ y teñidos con sales de plata (Sanguinetti et al., 1994). Los iniciadores para ISSR usados fueron: 899 (5'- CAC ACA CAC ACA-RG -3'), 901 (5'GTG TGT GTG TGT-YR -3') y 902 (5'- GTG TGT GTG TGT-AY -3'). Los fragmentos obtenidos fueron separados en geles de agarosa de alta resolución $1.5 \%$ y teñidos usando bromuro de etidio (Sambrook \& Russell, 2001).

Análisis de datos

Con las medidas obtenidas de las variables morfológicas se obtuvieron los parámetros de estadística descriptiva para cada una de las variables del fruto. Éstas fueron estandarizadas y posteriormente se construyó una matriz de 
correlación para llevar a cabo un Análisis de Componentes Principales. Con base en los dos primeros componentes principales se obtuvo un gráfico de dispersión. Para el análisis de las variables morfológicas se usó el programa NTSyS 2.11 (Rohlf, 2002).

Con base en el patrón de bandeo de los geles obtenidos para los marcadores ISTR e ISSR se construyeron matrices binarias de presencia-ausencia, dichas matrices fueron usadas para la detección del nivel de polimorfismo por loci y para determinar el coeficiente de similitud de Jaccard. Posteriormente se llevó a cabo un análisis de agrupamiento con el método UPGMA (Unweighted Pair-Group Method with arithmetic Averages). La estimación del coeficiente de similitud de Jaccard y el análisis de agrupamiento fueron llevados a cabo con el programa NTSyS 2.11 (Rohlf, 2002). Adicionalmente se llevó a cabo un análisis de remuestreo para confirmar la robustez de la tipología obtenida con un $95 \%$ de nivel de confianza y 10,000 repeticiones usando el programa Free Tree (Pavlicek et al. 1999). Por último la estructura genética de la población se estimó usando el programa STRUCTURE 2.3.1 (Pritchard et al., 2001), mediante un modelo que incluyó 5,000 iteraciones con un estimado de 2 a 5 grupos $(\mathrm{K}=2$ a $\mathrm{K}=5)$.

La comparación entre la información obtenida de la matriz de correlación y de la matriz de similitud (comparación de datos morfológicos y moleculares) se realizó mediante la prueba de Mantel (Mantel, 1967).

\section{Resultados y Discusión}

Caracterización morfológica y Análisis de componentes principales

De los caracteres evaluados, cinco presentaron valores mayores en coeficiente de variación. Los valores de estadística descriptiva se observan en el Cuadro 1.

\begin{tabular}{|c|c|c|c|c|c|c|}
\hline Variable & Media & $s$ & $S^{2}$ & Min. & Máx. & CV (\%) \\
\hline Diámetro del fruto $(\mathrm{cm})$ & 11.71 & 1.80 & 3.24 & 9.3 & 16 & 15.38 \\
\hline Longitud del fruto (cm) & 26.15 & 2.06 & 4.28 & 21 & 29 & 15.91 \\
\hline Número de semillas & 1.37 & 0.71 & 0.51 & 1 & 3 & 52.27 \\
\hline Peso del fruto (g) & 369.53 & 101.25 & 10252.39 & 235 & 705 & 25.53 \\
\hline Grosor lateral del mesocarpio $(\mathrm{cm})$ & 1.5 & 0.45 & 0.21 & 0.7 & 2.5 & 30.6 \\
\hline $\begin{array}{l}\text { Grosor longitudinal del mesocarpio } \\
(\mathrm{cm})\end{array}$ & 1.50 & 0.58 & 0.34 & 0.7 & 2.7 & 38.96 \\
\hline Forma del fruto & 2.06 & 0.85 & 0.74 & 1 & 3 & 41.40 \\
\hline
\end{tabular}

Los primeros tres componentes explicaron el $76.20 \%$ de la variación total. El poder discriminatorio de cada uno de los componentes principales estuvo en función de los valores propios de la matriz de correlaciones, los cuales se muestran en el Cuadro 2.

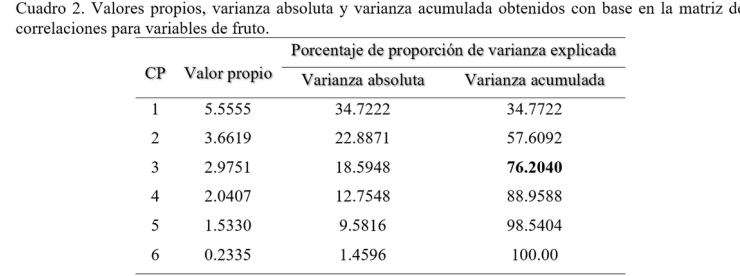

El componente principal 1 estuvo determinado por longitud del fruto, diámetro del fruto, longitud lateral del mesocarpio y longitud lateral del mesocarpio. El componente principal 2, por las variables de grosor lateral de mesocarpio, grosor longitudinal del mesocarpio y longitud del fruto. El componente principal 3, por su parte, por la longitud y el peso del fruto. El componente principal 4 se formó por la interacción de las variables de longitud del fruto, peso del fruto, diámetro del fruto, grosor lateral del mesocarpio y número de semillas.

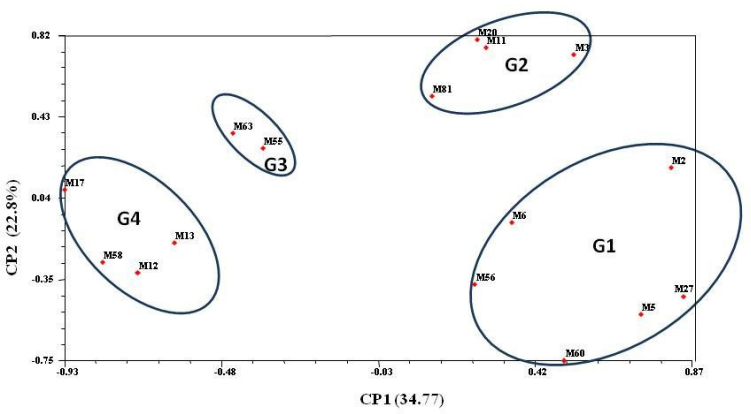

Figura 1. Gráfico de dispersión con base en los dos primeros componentes principales para las 15 muestras seleccionadas de la población de Colima. Entre paréntesis la cantidad en porcentaje de la variabilidad total explicada por cada uno de los componentes y abajo las variables que contribuyeron a la formación de cada componente.

Con base en los dos primeros componentes principales se construyó un gráfico de dispersión, en el que se distinguió la formación de cuatro grupos de frutos. El grupo 1 (G1) se conformó por las muestras: M2, M5, M16, M27, M56 y M60, que coincide con plantas del grupo I en el dendrograma (Figura 2); el grupo 2 (G2) contiene las muestras: M3, M11, M20 y M81; el grupo 3 (G3) por las muestras: M63 y M55; y el grupo 4 (G4) por las muestras: M12, M13, M17, M55, M58 y M63. Las características que contribuyeron en mayor medida a explicar la variabilidad total en el ACP fueron: longitud del fruto, peso del fruto, grosor longitudinal del mesocarpio, diámetro del fruto, numero de semillas y forma del fruto. Estos resultados concuerdan con los encontrados por Bayuelo-Jiménez (2007), Gaona-García (2008), IbarraEstrada (2012) y Arellano-Durán (2012), donde se mencionan que el análisis de las características que afectan las dimensiones del mesocarpio y la semilla, son de utilidad en la formación de grupos en el ACP. 


\section{Variabilidad detectada con ISSR}

En el análisis molecular con los dos marcadores se obtuvieron un total de 138 bandas, de las cuales 126 resultaron polimórficas, que representan un $91.3 \%$ de nivel de polimorfismo. Con el marcador ISTR fueron detectadas 102 bandas, 95 de las cuales fueron polimórficas, las cuales representan el $93.13 \%$ del total. Con el marcador ISSR se detectó la presencia de 36 bandas, de las cuales fueron polimórficas el $86.1 \%$. El elevado número de bandas polimórficas es indicativo de una elevada tasa de variabilidad genética dentro de la población, la cual se debe a que la totalidad de los individuos fueron propagados a partir de semilla, y a que no se ha realizado ningún trabajo de selección sobre el material analizado. Esto concuerda con lo encontrado en poblaciones de mamey provenientes de Guerrero y Veracruz, México por Ibarra-Estrada (2012), donde mediante el uso de RAPD y RAMP se encontraron porcentajes de polimorfismo que oscilan entre $75 \%$ y $92 \%$. Rodríguez-Rojas et al., 2012 por su parte reportan $82.4 \%$ de nivel de polimorfismo para muestras provenientes del estado de Morelos mediante el uso de RAPD.

El coeficiente de Jaccard mostró el valor máximo de similitud entre plantas colectadas en Yucatán con 0.94 y su valor mínimo con 0.33 entre individuos de la población de Colima.

El análisis de agrupamiento con el método UPGMA mostró la formación de cinco grupos en un punto de corte de 0.41. En estos grupos no se observó una separación clara entre plántulas y árboles adultos. Sin embargo, las ocho muestras provenientes de Yucatán formaron un grupo (grupo II) que se asoció al grupo principal de plantas de Colima (grupo I) con un nivel de 0.39 de de similitud. El resto de las plantas de Colima formaron tres grupos (grupos III, IV y V) que fluctuaron en nivel de similitud de 0.33 a 0.51 (Figura 6).

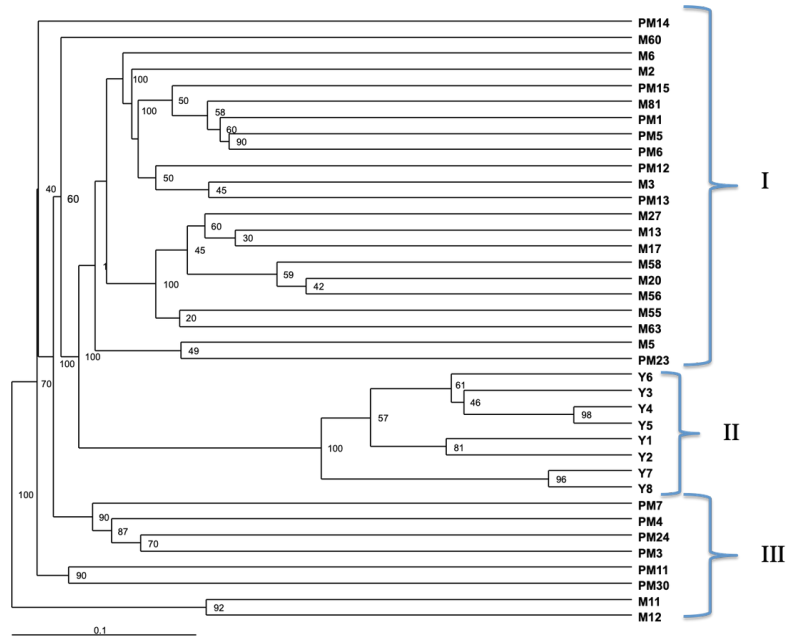

Figura 2. Dendrograma basado en la matriz de similitud de datos moleculares de mamey. Se muestra en los clados, el valor de bootstrap. Nomenclatura: $\mathbf{P M =}$ plántula, $\mathbf{M}=$ mamey $\mathbf{Y}=$ población de Yucatán

\section{Estructura genética}

Los resultados del análisis de estructura genética indican que la formación óptima fue de 5 grupos $(\mathrm{K}=5)$. Este número fue estimado con base en el valor más elevado de $\Delta \mathrm{K}$ (Cuadro 3 y Figura 3).

Cuadro 3. Valores de $\Delta \mathrm{K}$ para determinar el número óptimo de grupos genéticos formados en el análisis de estructura genética. En negritas se muestra el valor que determinó el número óptimo de grupos formados.

\begin{tabular}{cc}
\hline $\mathrm{K}$ & $\boldsymbol{\Delta} K$ \\
\hline 2 & - \\
3 & 0.946200 \\
4 & 2.409401 \\
5 & $\mathbf{5 . 7 8 6 7 6 1}$ \\
6 & - \\
\hline
\end{tabular}

Con base en los valores probabilísticos del análisis de estructura genética, se construyó un gráfico para cada una de las simulaciones realizadas ( $\mathrm{K}=2$ a $\mathrm{K}=5$ ) (Figura 4).

Para la población de Colima se puede apreciar que no existen componentes genéticos en común entre paltas adultas y plántulas. Los valores de Fst de cada grupo, el color que lo representa en el gráfico y heterocigosidad se muestran en el cuadro 4

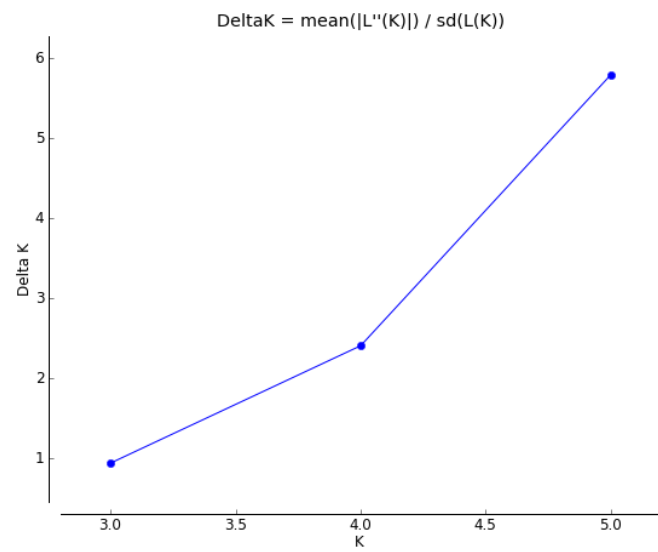

Figura 3. Grafico que muestra el número óptimo de grupos genéticos para las plantas de Colima (K=5) según el método de Evanno y cols (2005).

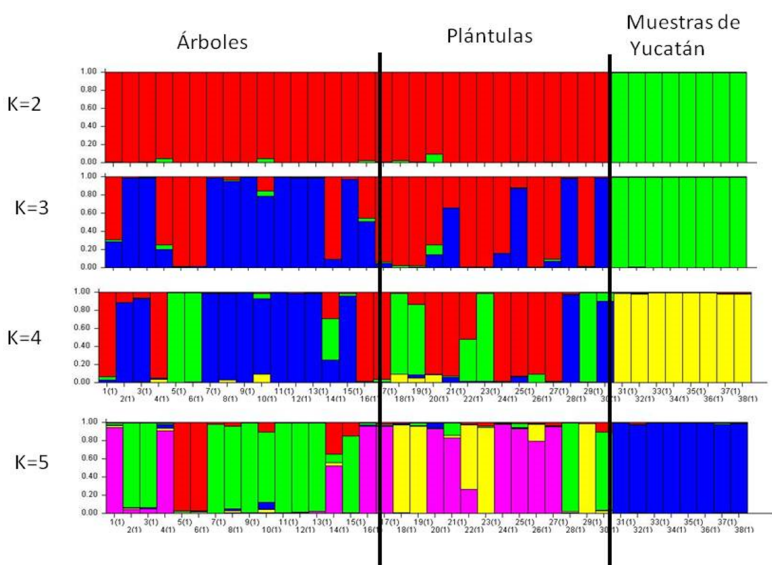

Figura 4. Representación gráfica de cada una de las simulaciones probabilísticas realizadas con el programa STRUCTURE. El número de grupos es equivalente al número de colores en cada gráfico. La probabilidad de pertenecer a cada grupo aparece indicada en el eje $\mathrm{Y}$, las muestras en el eje X. 
Cuadro 4. Valores de diferenciación genética (Fst) para cada uno de los grupos obtenidos usando el programa STRUCTURE. Los valores son provenientes del cálculo en $\mathrm{K}=5$

\begin{tabular}{ccc}
\hline Color & Diferenciación genética (Fst) & Hetrocigosidad (HE) \\
\hline Rojo & 0.0747 & 0.2291 \\
Verde & 0.5818 & 0.3671 \\
Azul & 0.1014 & 0.1921 \\
Amarillo & 0.0015 & 0.3361 \\
Rosa & 0.2277 & 0.3669
\end{tabular}

El análisis llevado a cabo con el programa STRUCTURE muestra además que existe un alto grado de diferenciación entre las muestras colectadas en Colima y las muestras colectadas en Yucatán, por lo que se descarta que exista una relación de procedencia de las muestras de Colima con respecto a las de Yucatán. El análisis de estructura genética de la población mostró además que no hay un componente genético común que sea claramente visible para las muestras de Colima, sin embargo existen componentes genéticos característicos si las muestras son separadas entre árboles adultos y plántulas, lo que sugiere que existe un grado considerable de autopolinización entre los individuos que componen la población.

La heterocigosidad, es difícil de interpretar cuando se utilizan marcadores dominantes como los utilizados en el presente trabajo (Katarzyna y cols., 2013) sin embargo, al calcularla, se puede hacer referencia al estado de variabilidad entre los grupos simulados por el programa STRUCTURE. Los valores de heterocigosidad calculada, son relativamente bajos (Cuadro 4) y revelan un grado alto de autofecundación en esta especie, sobre todo en los individuos marcados con azul y rojo que mostraron los menores valores de HE. Estos niveles de heterocigosidad encontrados, apoyan el perfil genético reportado en los anteriores resultados. Estos resultados concuerdan con lo reportado por Martínez-Castillo et al. (2019).

\section{Conclusiones}

Como resultado del análisis llevado a cabo en mamey, se puede concluir que ambos marcadores moleculares fueron capaces de producir una cantidad considerable de bandas polimórficas que fueron informativas para el estudio. Los niveles de variabilidad encontrados en este y otros trabajos para poblaciones de mamey cultivadas fuera y dentro de la zona de origen sugieren que es necesario llevar a cabo estudios sistemáticos que incluyan un mayor número de marcadores moleculares así como una mejor representación de las poblaciones cultivadas con la finalidad de tener un panorama más claro de la diversidad genética de esta especie.

\section{Literatura Citada:}

- Azurdia, C. 2006. Tres especies de zapote en América Tropical. Southampton Centre for Underutilised Crops, University of Southampton. Southampton

- Arcos-López, E. 2011. Caracterización del Sistema de Producción de Mamey (Pouteria sapota Jacq.) del municipio Huamuxtitlán, Gro. Tesis para obtener el grado de: Maestro Tecnólogo. Colegio de Postgraduados, Universidad Autónoma Chapingo

- Arellano-Durán, N. 2012. Caracterización morfológica, estudio molecular y determinación de azúcares en zapote mamey (Pouteria sapota (Jacq.) H.E. Moore \& Stearn). Tesis para obtener el grado de: Maestro en Ciencias. Colegio de Postgraduados, Universidad Autónoma Chapingo

- Bayuelo-Jiménez, J.S. y I. Ochoa. 2006. Caracterización morfológica del zapote mamey [Poueria sapota (Jacq.) H.E. Moore \& Stearn] en el centro occidente de Michoacán, México. Revista Fitotecnia Mexicana. 29 (1): 9-17.

- Castellanos-Hernández, O. A., R. Lepiz-Ildefonso, G. E. Castellanos-Enríquez, A. Rodríguez-Sahagún y M. I. Torres-Morán. 2017. Relaciones genéticas basadas en marcadores ISTR entre formas silvestres, cultivadas e intermedias de frijol de guía colectado en Jalisco, México. Acta Botanica Mexicana. 118: 53-63. DOI: http://dx.doi.org/10.21829/abm118.2017.1200

- Carrara, S., R. Campbell y R. Schnell, 2004. Genetic Variation among cultivated selections of mamey sapote (Pouteria spp. [Sapotaceae]). Proceedings of the Florida State Horticulture Society. 117: 195-200

- Elias-Nascimiento, V., A.B. Geraldo-Martins y Hassayuki Hojo. 2008. Caracterização física e química de frutos de mamey. Revista Brasileira de Fruticultura. 30(4): 953-957.

- Espinos, Z., C. Saucedo, A. Villegas y M.E. Ibarra. 2005. Caracterización de frutos de zapote mamey en Guerrero, México. Interamerican Society for Tropical Horticulture. 48: 135-138.

- Gaona-García, A., I. Alia-Tejacal, V. López-Martínez, M. Andrade Rodríguez, M.T. Colinas-León y O. Villegas Torres. 2008. Caracterización de frutos de zapote mamey (Pouteria sapota (Jacq.) H.E. Moore \& Stearn) En el sureste del Estado de Morelos. Revista Chapingo Serie Horticultura. 14 (1): 41-47

- Gómez-Jaimes, R., D. Nieto-Aguilar, D. Téliz-Ortiz, J.A. Mora-Aguilera, C. Nava Díaz, M.T. MartínezDamián y M. Vargas-Hernández. 2012. Manejo postcosecha del zapote mamey (Pouteria sapota (Jacq.) H.E. Moore \& Stearn) y su impacto en la calidad de la fruta. Revista Chapingo, Serie Horticultura. 28 (2): 253262

- Ibarra-Estrada, M.E. 2012. Morfología de frutos, perfil de ácidos grasos en semilla y diversidad genética 
molecular en selecciones de zapote mamey (Pouteria sapota (Jacq.) H.E. Morre \& Stearn). Tesis para obtener el grado de: Maestro en Ciencias. Colegio de Postgraduados, Universidad Autónoma Chapingo

- Infante D, Molina S, Demey JR, Ga'mez E (2006) Asexual genetic variability in Agavaceae determined with inverse sequence tagged repeats and amplification fragment length polymorphism analysis. Plant Mol Biol Rep 24:205-217

- Katarzyna, B., N. Patterson y D. Reich. 2013. A Novel Approach to Estimating Heterozygosity from LowCoverage Genome Sequence. Genetics, (195): 553-561.

- Keb-Llanes, M., G. González, B. Chi-Manzanero y D. Infante. 2002. A rapid and simple method for small scale DNA extraction in Agavaceae and other tropical plants. Plant Molecular Biology Reporter. 20: 299

- Mantel, N. (1967). The Detection of Disease Clustering and Generalized Regression Approach. Cancer Research. 27 (2): 209-220.

- Martínez-Castillo, J., N.H. Blancarte-Jasso, G. ChepeCruz, N.G. Nah-Chan, M.M. Ortíz-García y R.S. Arias. (2019). Structure and genetic diversity in wild and cultivated populations of Zapote mamey (Pouteria sapota, Sapotaceae) from southeastern Mexico: its putative domestication center.

- Nava-Cruz, Y. y Ricker, M. 2004. El Zapote Mamey [Pouteria sapota (Jacq.) H.E. Moore \& Stearn] un fruto de la selva mexicana con alto valor comercial. En: Alexiades, M.N. y P. Shanley, Eds. 2004. Productos Forestales, medios de subsistencia y conservación: Estudios de Caso sobre sistemas de manejo de productos forestales no maderables. Vol. 3. Centro para la Investigación Forestal Internacional. Indonesia

- Pavlicek, A., S. Hdrá y J. Flegr. 1999. Free-Tree: Freeware program for construction of Phylogenetic trees on the basis of distance data and bootstrap/jackknife analysis of the tree robustness. Application in the RAPD analysis of the genus Frenkelia. Folia Biologica. 45 (3): 79.

- Pritchard, J.K., M. Stephens y P. Donnelly. 2000. Inference of population structure usin multilocus genotype data. Genetics. 155: 945-959

- Rodríguez-Rojas, T.J., M. Andrade-Rodríguez, I. AliaTejacal, V. López-Martínez, S. Espinoza-Zaragoza y H. Esquinca-Avilés. 2012. Caracterización molecular del zapote mamey (Pouteria sapota (Jacq.) Moore \& Stearn). Revista de la Facultad de Agronomía LUZ. 29: 339-354.

- Rohlf, J.2002. Introduction to the exploration of multivariate biological data. Quarterly Review of Biology. 77: $50-51$

- Sambrook, J. y D.W. Russell. 2001. Molecular Cloning: A laboratory manual. 3 vol. Cold Spring Harbor Laboratory Press. New York
- Sanguinetti, C.J., E. Díaz-Neto y A.J. Simpson. 1994. Rapid silver staning and recovery of PCR products separated on polyacrilamide gels. Biotechniques. 17: 14-21

- SIAP. 2018. Anuario estadístico de la producción agrícola en 2017. https://www.gob.mx/siap/ acciones-yprogramas/producción-agrícola-33119. Consultado en Dic 2018.

- Torres-Morán, M.I., Almaraz-Abarca N., VelascoRamírez A.P., Hernández-Vargas V., Orea-Lara G., Cifuentes-Díaz de León A., Oliver-Salvador C. (2008) Taxonomic significance of ISTR to discriminate species in Agavaceae. Am J Agric Biol Sci 3:661-665.

- Villegas, M.A. y A. Mora. 2008. Propagación del zapote mamey (Pouteria sapota (Jacq.) H.E. Moore \& Stearn). En: El zapote mamey en México: avances de investigación. Alia, T., A. Villegas, V. López, M. Andrade, M. Acosta, O.G. Villegas y D. Guillén. Universidad Autónoma de Morelos. Cuernavaca. 